\title{
Advanced Security System using Daugman's Model for IRIS Authentication
}

\author{
Arti Patil \\ Student, M.E. (Computer Engineering) \\ St. Francis Institute of Technology, \\ University of Mumbai, \\ Mumbai - 400103, India.
}

\author{
Deepak Jayaswal, $\mathrm{PhD}$ \\ Prof. Dept. of Electronics and \\ Telecommunication Engg., \\ St. Francis Inst. of Technology, \\ Mumbai - 400103, India
}

\begin{abstract}
In world, insecurity increases day by day, so there is a need for authentication which is more essential and challenging. Iris Recognition is one of the ways to secure authentication because of uniqueness and robustness. Human iris is one of the parts in the human body, which develops a unique pattern before birth, have produced very high rates of perception. But, still there are some challenges in iris authentication like Countering the effect of light reflection on images, if a person is suffering from light reflections on eye images affects the iris detection so that original information of this part of the image has been lost and also there is an observable difference in intensity values between this 'light affected region' and it's surrounding darker region. There is effect on iris which creates problem in accurate detection, In order to avoid light reflection affecting the iris detection, a morphological operator is used. Localization of the iris borders in a watch image will be thought of as a significant step within the iris recognition method. There exist several algorithms to phase the iris. one amongst the segmentation ways, that's employed in several industrial iris biometric systems is associate rule called a Daugman's rule. The aim of this paper is to implement this rule victimization MATLAB programming atmosphere.
\end{abstract}

\section{Keywords}

Iris recognition, Daugman's Model, morphological operator

\section{INTRODUCTION}

Biometric authentication has become increasingly popular in security systems. This Systems provide an automatic recognition based on different unique features or characteristics. Various biometric systems are developed on fingerprints, facial features, voice, Hand geometry, Handwriting, the retina and the one presented in this paper, the IRIS. The iris is so reliable as a form of identification because of the distinctiveness of its pattern. Although there's a genetic influence, notably on the iris color, the iris develops through folding of the tissue membrane then degeneration (to produce the pupil opening) which ends during a random and distinctive iris [2].

\subsection{Introduction Of Eye Image}

Eye is created from 5 elements like palpebra(eyelid), pupil, limbus, iris, and sclera. Iris is that the one a part of eye. Iris could be a skinny, circular structure within the eye that to blame for dominant the diameter and size of the pupil.

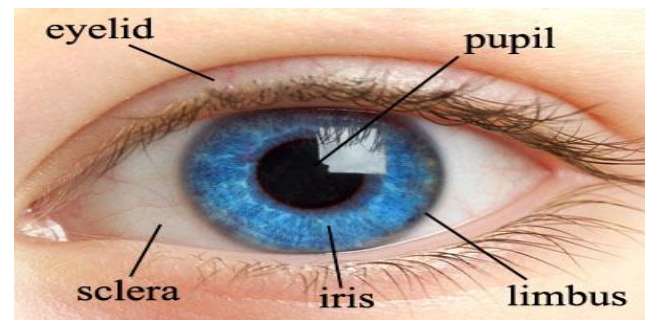

Fig 1. Human Eye Anatomy.

\subsection{Iris Recognition}

Iris Recognition could be a chop-chop increasing technique of biometric identification that uses pattern-recognition techniques on pictures of irises to unambiguously determine a personal[6].Iris recognition is that the method of recognizing someone by analyzing the random pattern of the iris Fig. one shows the anatomy of human eye that contain the world of albuginea and iris for references. The iris image has to be extracted from the initial eye image. Iris localization could be a method to isolate the iris region from the remainder of the noninheritable image. Iris is approximated by 2 circles, one for iris/sclera boundary and another for iris/pupil boundary. The iris biometric deals with distinguishing a person's being by his/her iris pattern extracted from the pictures of his/her eye. As shown in Figure one, the human eye consists of three major parts: pupil (the innermost black part), iris (the coloured part) and albuginea (the white part). The iris and pupil as aforesaid to be nonconcentric [2]. The radius of inner border of the iris i.e. it's border with the pupil is additionally not constant since the scale of pupil will increase and reduces looking on the number of sunshine incident to the pupil.

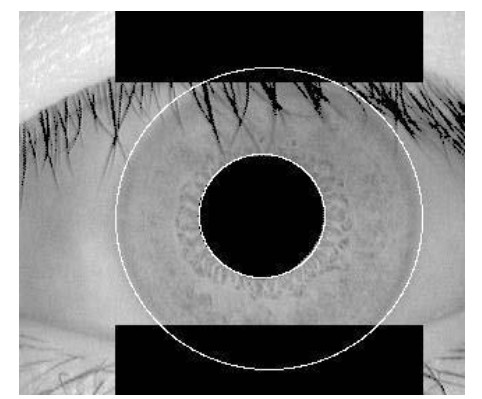

Fig 2. Iris and Pupil detection of eye

Each individual incorporates a distinctive pattern of iris [2]. This pattern is extracted from the image of the attention and encoded. 


\section{REVIEW OF LITERATURE}

Human iris possesses genetic independence and contains very information-rich body and distinctive texture pattern that makes it extremely complicated enough to be used as a biometric signature. applied math analysis reveals that the iris is that the most mathematically distinctive feature of the physical body due to the many degrees of freedom it offers with the power to accurately live its texture [1]. Reliable biometric verification and identification techniques based upon iris patterns are given by general [2], Wildes et al. [5-7], Boles [8,9]. different bestknown iris recognition systems are introduced by Zhu et al. [10], Lim et al. [11], Noh et al., Tisse et al. [6] and Ma et al. [11]. impelled by these works, many researchers worked on enhancing the performance of iris recognition systems. Some researches specialise in rising the image acquisition systems, some deals with enhancing the segmentation algorithms, others square measure dedicated to rising the options extraction and encryption method. In life science generally, it's been found that victimisation multiple images for enrollment and comparison the probe to multiple gallery samples can end in improved performance [8]. author and Wildes [3-4] and Martin- Roche et al. used integro-differential operators that search over tridimensional areas, having as goal the maximization of equations that localize each iris borders. Wildes [12] started the segmentation of the iris ring by the development of a binary edge-map. Next, used the circular Hough rework to suit circles that delimit the iris ring. this is often the foremost sometimes seen methodology within the iris segmentation literature and is planned with minor variants by Wang and associates [11-12]. conjointly based mostly in Wildes, Proença and Alexandre planned a technique that uses a clump method to extend the strength to shrie knowledge. The method planned by Du et al. is predicated on the previous detection of the pupil. The image is then reworked into polar coordinates and therefore the iris outer border localized because the largest horizontal edge resultant from Sobel filtering. This approach might fail just in case of non-concentric iris and pupil, and of terribly dark iris textures. caustic WI Liam, Ali Chekima, Liau Chung Fan and Jamal Ahmad Dargham, in 2002,proposed [1] a system consisting of 2 parts: Localizing Iris and Iris Pattern Recognition. They used photographic camera for capturing image; from the captured pictures Iris is extracted. solely the portion of selected Iris then reconstructed into parallelogram format, from that Iris pattern is recognized. Weiki Yuan, Zhonghua architect and lutecium Xu have analyzed eye pictures [9] that they need supported structure characteristics of eyes, they recommend a speedy iris location arithmetic. Firstly, they need got Associate in Nursing approximate center by grey projection, have gotten 2 points that set at left and right boundary by threshold worth severally, and have gotten a degree that set at the lower boundary by direction edge detection operators, then they ensured the boundary of pupil and probable center. Secondly, they need got actual pupil boundary and center by Hough rework that's processed at alittle scope close the probable center. Thirdly, they need searched 2 points that set at left and right boundaries between iris and sclerotic coat on horizontal direction by victimisation the precise center and direction edge detection operators. Then they ensured the horizontal coordinate of the middle of iris supported the higher than 2 purpose accurately. Finally, they need searched 2 points that set at higher and lower boundaries between iris and sclerotic coat starting at the horizontal coordinate of the middle of iris on the directions that creating and and minus thirty angles between horizontal direction severally by victimisation direction edge detection operators, so that they ensured the coordinate of the middle of iris and therefore the boundary between iris and sclerotic coat.

\section{PROPOSED WORK}

To with success perform iris recognition, correct iris segmentation may be a important requirement.Two wide used and renowned strategies of iris segmentation area unit Wilde's technique and Daugman's technique severally. Oscar Fingal O'Flahertie Wills Wilde has projected a two step iris segmentation method: 1) a binary edge map supported gradient primarily based is built from the intensities of the pixels in associate degree iris image, 2) the iris inner and outer borders area unit detected victimization Hough remodel [9].

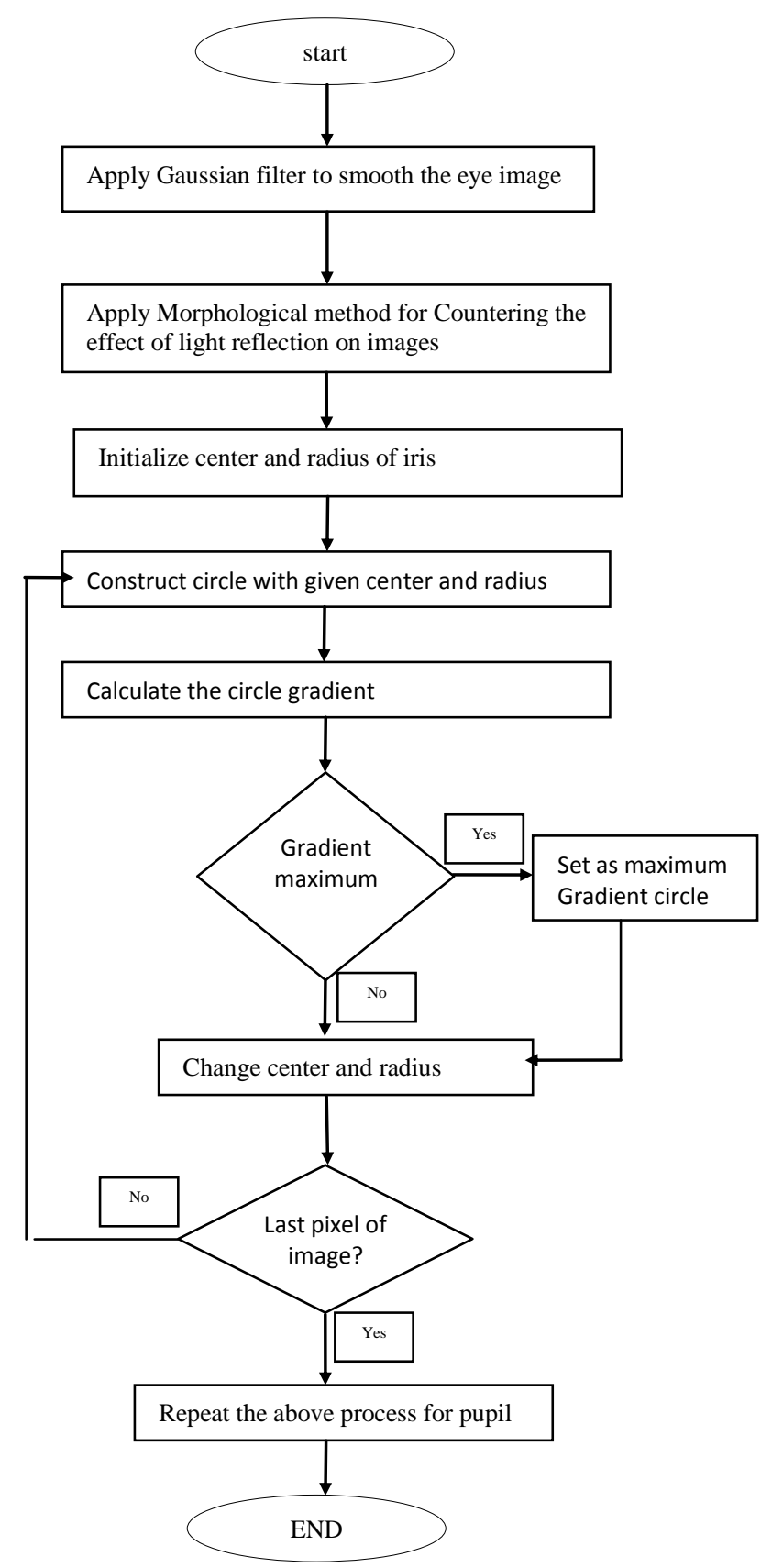

Fig 4: Flow chart describing Daugman's method And Morfological Method.

Daugman's rule, named once the academician John Daugman, is associate degree integro-differential operator that searches over a picture of the attention for the circular pupil and also the bodily structure borders of the iris. it's a circular edge detector that searches for the parameters of a circular border. The total of circumferential constituent intensity values at this circular border 
ought to have the most amendment in values compared to a one constituent radius wider circular region removed from an equivalent center constituent, as for the circular border. Daugman's rule for iris segmentation is employed during this paper.

\subsection{The Enhancement Of An Eye Image Before The Application Of The Daugman's Operator}

- Countering the effect of light reflection on image: -

Light reflections within the eye pictures negatively have an effect on iris border detection by suggests that of Daugman's rule. The white spot in Figure three and also the 'white region' indicated by the dotted blue circles show the sunshine reflections within the eye pictures. These light-weight reflections cowl the components of the attention image inflicting hindrance within the iris detection method. As are often determined, the 'light reflection affected region' may be a region consisting of the pixels with high intensity values (since it's a white region). The region close it's an area consisting of the pixels with low intensity values (since it's a darker region). This component of sunshine reflections on eye pictures affects the iris detection in 2 ways in which. Firstly, the initial constituent intensity values are replaced by the upper intensity values within the a part of the image, suffering from light-weight reflection thus the initial data of this a part of the image has been lost. Secondly, there is associate degree discernible distinction in intensity values between this 'light affected region' and it's close darker region. During this paper morphological technique applied to such pictures detects the intensity amendment at this reflection affected white regions to be the most amendment in total of circumferential pixels intensity values. thus because of this component of sunshine reflection the iris and pupil borders area unit incorrectly known.

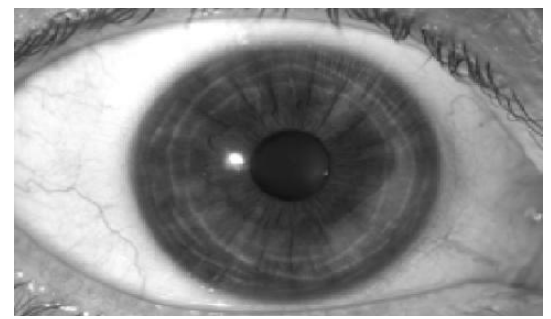

Fig 3: Light reflection affected eye image

\subsection{Daugman's operator:}

The task is to seek out the centre coordinates and therefore the radius of the iris and therefore the pupil and Daugman's equation is utilized for this task. The centre purpose of Daugman's theory of border recognition is that the integrodifferential equation as follows,

$\max \left(r, x_{0}, y_{0}\right)\left|G_{\mathrm{c}}(r) * 2 / \partial r_{(r, x 0, y 0)} \S \mathrm{I}(\mathrm{x}, \mathrm{y}) / 2 \Pi r\right| d s$

$\mathrm{I}(\mathrm{x}, \mathrm{y})$ is that the intensity of the picture element at coordinates $(\mathrm{x}, \mathrm{y})$ within the image of an iris. $\mathrm{r}$ denotes the radius of assorted circular regions with the middle coordinates at $(\mathrm{x} 0, \mathrm{y} 0) . \sigma$ is that the variance of the Gaussian distribution.G $\sigma(r)$ denotes a mathematician filter of scale letter $(\sigma) .(\mathrm{x} 0, \mathrm{y} 0)$ is that the assumed centre coordinates of the iris. $\mathrm{s}$ is that the contour of the circle given by the parameters $(\mathrm{r}, \mathrm{x} 0, \mathrm{y} 0)$.

\subsection{Two dimensional 2D Gaussian filter:}

In physics and signal process, a mathematician filter may be a filter whose impulse response may be a mathematician operate. Mathematically, a mathematician filter modifies the signal by convolution with a mathematician operate. Some of the mathematician sleeking operator may be a 2-D convolution operator that's wont to smooth the photographs of the attention so as to get rid of noise.The one-dimensional mathematician filter has an impulse response given by

$$
g(x)=\sqrt{\frac{a}{\pi}} \cdot e^{-a \cdot x^{2}}
$$

where ' $a$ ' denotes the peak of the curve's peak (magnitude of the impulse response) and features a worth bigger than zero. The reduction of the number of object pixels, on which the Daugman's operator is applied, makes the iris detection process faster.

\section{RESULT}

\subsection{Result of Light reflection affected eye image}

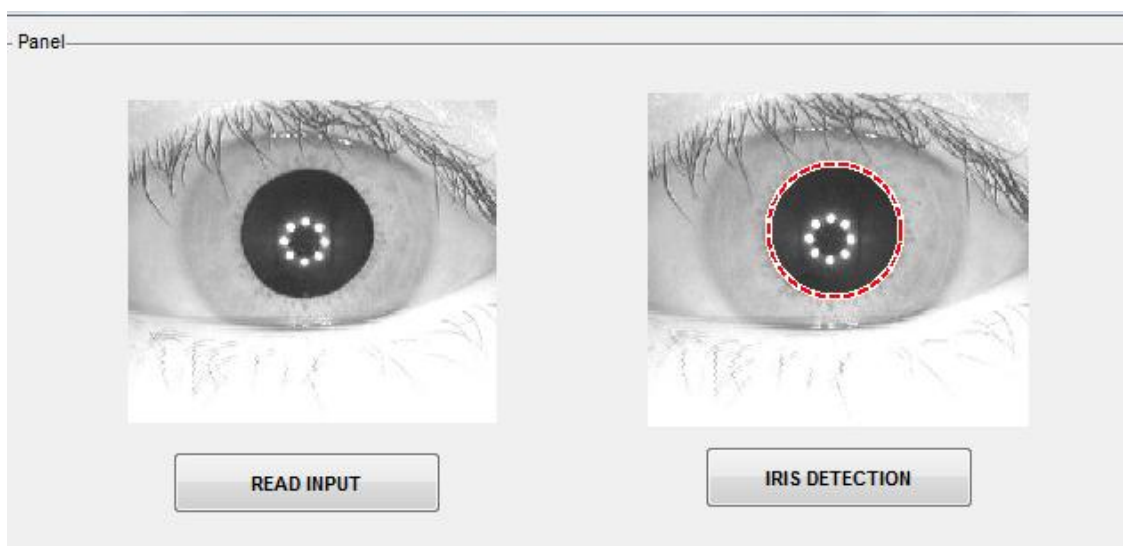




\subsection{Result of valid eye image in the database}

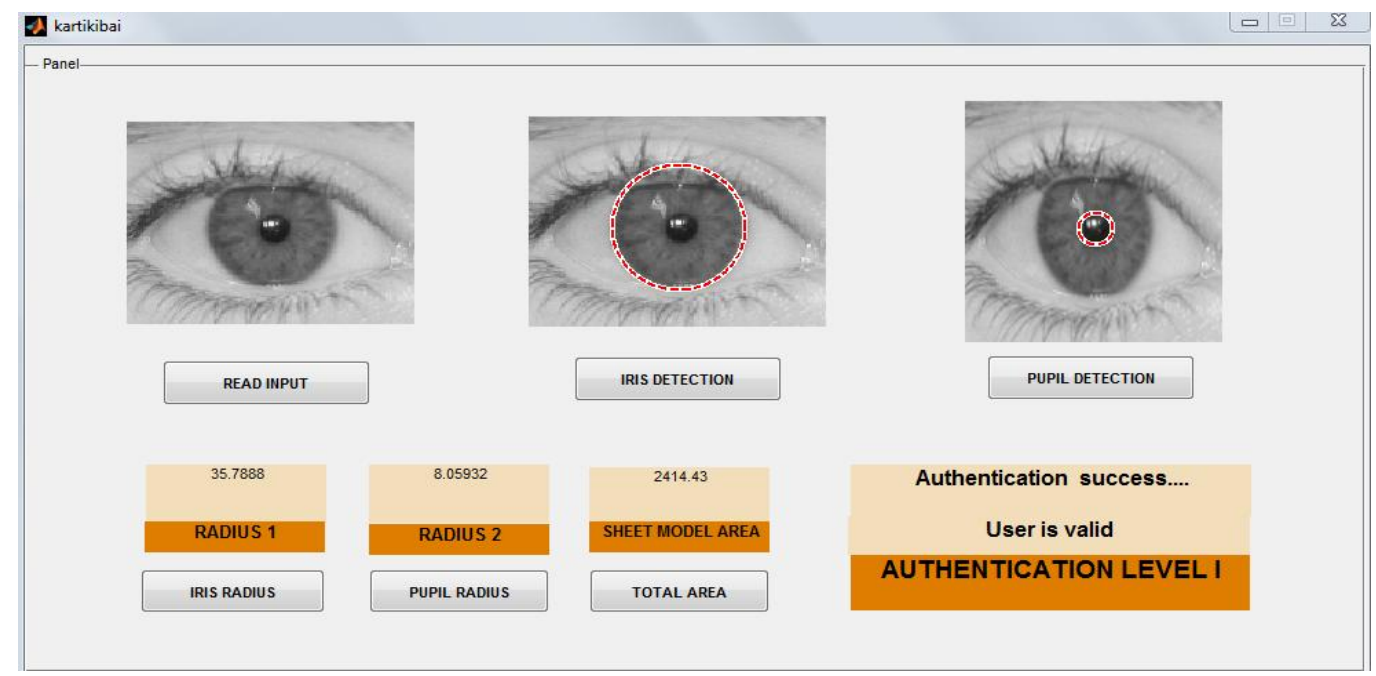

\subsection{Result of invalid eye image}

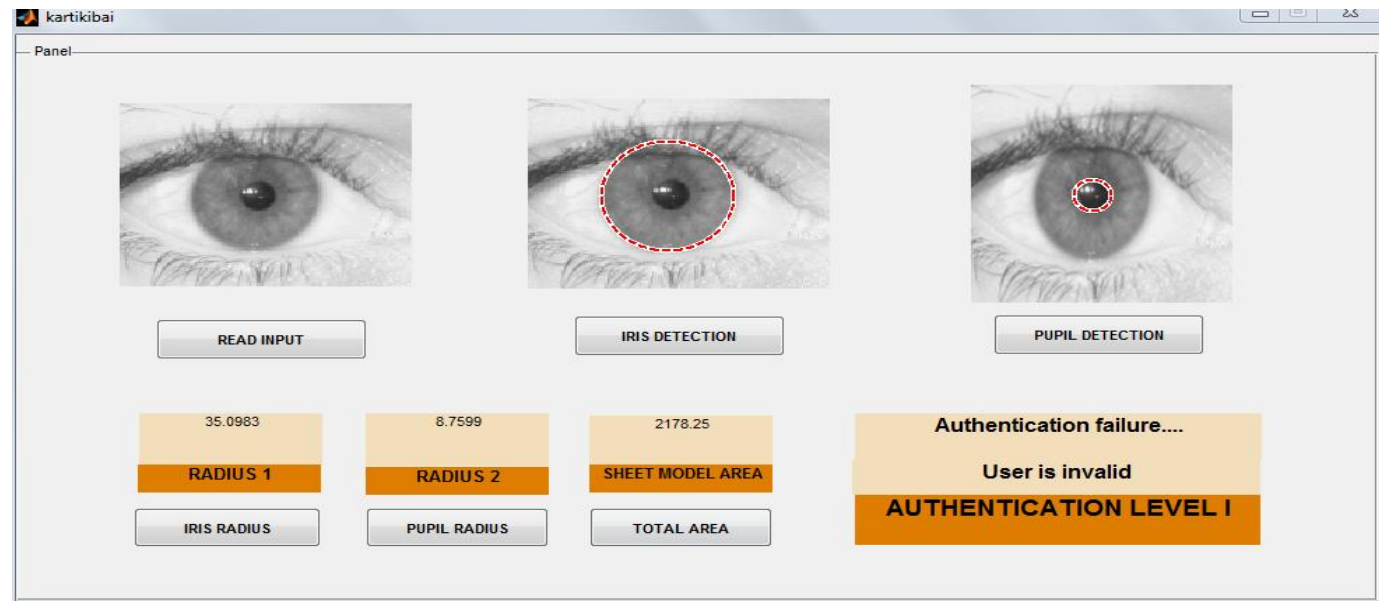

\section{CONCLUSION}

Review of this paper, summarizes the problems and challenges with current iris biometric systems. specially, we have a tendency to mentioned security and performance connected problems.In this paper address the security problem using daugman's method. we've shown that a lot of fashionable beliefs concerning security, reliableness, stability and performance of iris recognition systems don't seem to be correct and wish to be revisited. The problems raised during this survey ought to be self-addressed so as to be a lot of assured in operating of iris recognition systems. The importance of iris authentication system with the help of new approach is very high. A totally new business model can be developed with the help of current approach. The current system has been developed with the help of matlab programming language and some Mathematical operations, it is performing according to our expectations butthere are some limitations such as blur and dynamically taken images. In future, we have a tendency to conceive to explore the safety and privacy issues facing alternative biometric systems. This could result in a style of multi-biometric system that overcomes the weaknesses of one by the strength of alternative biometric.

\section{REFERENCES}

[1] Prof. Bharti L. Dhote1, Mr. Abhijit Kedari2,Miss. Shashanki Deshmane,Miss. Anuradha Gaikwad "Iris Recognition System for Secure Authentication",IJETSTVolume ||01||Issue ||03||Pages 264-267||May||ISSN 234894802014.

[2] Nathan D. Kalka, Jinyu Zuo, Natalia A. Schmid, Bojan Cukic Lane, "Image quality assessment for iris", Department of Computer Science and Electrical Engineering, West Virginia University,Morgantown, WV 26506, USA.

[3] Kaushik Roy*, Darrel Hudgin*, Prabir Bhattacharya* and Ramesh Chandra Debnath $\dagger$ "Iris Recognition:A Java based Implementation” 1-4244-1551-9/07/\$25.00 (02007 IEEE.

[4] Li Ma, Tieniu Tan, Fellow "Efficient Iris Recognition by Characterizing Key Local Variations," IEEE,Yunhong Wang Member, IEEE, andDexin Zhang Elsevier J. on Computers in Industry 57 (2006) pp. 622-630.

[5] John Daugman. Recognizing persons by their iris patterns. Cambridge University Cambridge,UK. 2001. 
[6] Kevien W. Boyer,Karen Hollingsworth,"Image understanding for iris biometrics:A survey",@2007 Elsevier.

[7] Michael Boyd,Dragos Carmaciu,Francis Giannaros,Thomas Payne,William Snell," Iris Recognition” ISSN 1989-1660 March 19, 2010

[8] RICHARD P. WILDES,'Iris Recognition:An Emerging Biometric Technology", PROCEEDINGS OF THE IEEE, VOL. 85, NO. 9, SEPTEMBER 1997.

[9] Sajida Kalsoom, Sheikh Ziauddin," Iris Recognition: Existing Methods and Open Issues", PATTERNS 2012 : The Fourth International Conferences on Pervasive Patterns and Applications.
[10] V.Larrumbe Hidalgo, L.Martin Garcia, M.Taboada "Iris recognition using the JAVAVis Library," International Journal of Interactive Multimedia and Artificial Intelligence, Vol. 1, N 1, ISSN 1989-1660,2008

[11] Upasana Tiwari, Deepali Kelkar,Abhishek Tiwari,"Study of Different IRIS Recognition Methods" IJCTEE Volume 2, Issue 2009.

[12] Sachin Gupta Assistant Professor, Karnal, Haryana Ashish Gagneja Associate Professor,"Proposed Iris Recognition Algorithm through Image Acquisition technique" (C) 2014, IJARCSSE All Rights Reserved. 